\title{
Upper Lip Swelling-An Uncommon and Avoidable Complication Following Cerebellopontine Angle Tumor Excision Requiring Transcranial Facial Motor-Evoked Potential Monitoring
}

\author{
Ramamani Mariappan ${ }^{1}$ Ishaan Suniara ${ }^{1}$ Baylis V. Joseph² Srinivasa B. Krothapalli³
}

${ }^{1}$ Department of Neuroanaesthesia, Christian Medical College, Vellore, Tamil Nadu, India

2Department of Neurological Sciences, Christian Medical College, Vellore, Tamil Nadu, India

${ }^{3}$ Neurophysiology Unit, Department of Neurological Sciences, Christian Medical College, Vellore, Tamil Nadu, India

J Neuroanaesthesiol Crit Care 2022;9:60-61.

A 45-year-old hypertensive male on nifedipine and atenolol was diagnosed with a left cerebellopontine (CP) angle tumor $(4.0 \times 4.4 \times 4.5 \mathrm{~cm})$, with features of raised intracranial pressure. He underwent left retromastoid suboccipital craniotomy and excision of the tumor with transcranial facial motor-evoked potential (Tc-MEP) monitoring. On the day of surgery, after attaching all the standard monitors, arterial blood pressure, and bispectral index (BIS), induction was performed with propofol $(2 \mathrm{mg} / \mathrm{kg})$ and fentanyl $(2 \mathrm{mcg} / \mathrm{kg})$ and paralyzed with vecuronium $(0.1 \mathrm{mg} / \mathrm{kg})$. Anesthesia maintenance was performed with total intravenous anesthesia (TIVA), using propofol $(100-150 \mathrm{mcg} / \mathrm{kg} / \mathrm{min})$ and fentanyl infusion $(1-2 \mathrm{mcg} / \mathrm{kg} / \mathrm{hr})$, to facilitate facial MEP monitoring. As part of the MEP protocol, a single thick bite block (rolled-up gauze piece) was inserted in the midline between the upper and lower jaw, with adequate space available between the two. A ring finger was inserted into the oral cavity between the tongue and the bite block to ensure there was no tongue folding. The patient was positioned in the right lateral position, and the surgery proceeded without any undue incidents. Facial MEP monitoring (to assess the corticobulbar tract) was performed via $\mathrm{C} 1 / \mathrm{C} 2$ motor zone transcranial electrical stimulation (electrode placed $1 \mathrm{~cm}$ anterior to $\mathrm{C} 1 / \mathrm{C} 2$ over the motor cortex area, called M1/M2), using $180 \mathrm{~V}$ stimulation threshold current. Stimulation was performed 30 times during the surgery, and the response was excellent. His intraoperative period was stable without hemodynamic fluctuations. Subtotal excision was done, leaving behind a 5-mm tumor margin on the brain stem and the facial nerve. The surgery lasted for 8 hours with $1200 \mathrm{~mL}$

published online October 24, 2021
DOI https://doi.org/

$10.1055 / \mathrm{s}-0041-1732829$ ISSN 2348-0548
Address for correspondence Ramamani Mariappan, MD, Department of Neuroanaesthesia, Christian Medical College, Vellore 632004, Tamil Nadu, India (e-mail: ramamani@ cmcvellore.ac.in).

blood loss, and a unit of blood was transfused. At the end of the surgery, the patient was turned supine, and the endotracheal tube was taken out after meeting the extubation criteria. Lignocaine (preservative-free) and labetalol were used to control the extubation response. After extubation, while removing the bite block, we noticed that the inner upper lip mucosa $(0.5 \mathrm{~cm})$ was caught between the upper incisor and the bite block. A bite mark was noted on the mucosal surface, and there was no swelling or laceration noted at that point in time. As localized lip swelling was anticipated, ice pack was placed, and topical steroid cream was applied to the mucosal surface and upper lip and continued during the postoperative period. The next day, massive upper lip swelling ( $\mathbf{- F i g . ~ 1 A )}$ was noted, which was tense and painful. Since it involved the "dangerous area of the face," an urgent dental consultation was sought, and emissary vein thrombosis was ruled out. It was managed conservatively with topical steroid cream and antibiotics (Augmentin and Flagyl). The lip swelling gradually came down after 8 days but was not normalized ( - Fig. 1B) at the time of discharge. The patient was reassured and advised to follow-up.

The indications for the use of Tc-MEP monitoring have expanded over the past decade. In our institution, the use of facial MEP to assess the integrity of the corticobulbar tract has become routine in patients undergoing $\mathrm{CP}$ angle tumor excision. Tc-electrical stimulation can cause bite injuries; although it is uncommon, it is a disturbing complication with an incidence of $0.63 \%$. The incidence of severe injuries requiring sutures is $0.14 \% .^{1}$ Tongue injuries are more common compared with lip injuries $(4: 1){ }^{1}$ (c) 2021. Indian Society of Neuroanaesthesiology and Critical Care. All rights reserved.

This is an open access article published by Thieme under the terms of the Creative Commons Attribution-NonDerivative-NonCommercial-License, permitting copying and reproduction so long as the original work is given appropriate credit. Contents may not be used for commercial purposes, or adapted, remixed, transformed or built upon. (https://creativecommons.org/licenses/by-nc-nd/4.0/).

Thieme Medical and Scientific Publishers Pvt. Ltd. A-12, 2nd Floor, Sector 2, Noida-201301 UP, India 


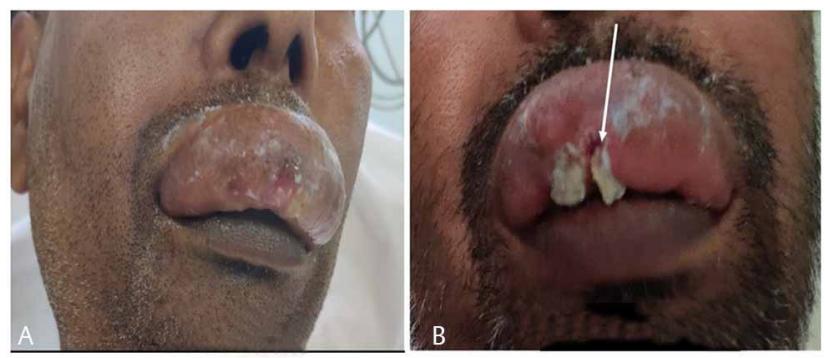

Fig. 1 (A) Upper lip swelling 12 hours after surgery. (B) Regression of upper lip swelling 7 days after surgery; the arrow is showing the site at which the mucosa was caught between the teeth and bite block.

In this case, after applying the bite block, while ruling out tongue folding, the inner upper lip mucosa was caught between the bite block and the upper incisor, which went unnoticed and led to this complication. This avoidable injury led to patient discomfort, use of antibiotics, and increased length of hospital stay along with financial implications. In our case, prolonged entrapment of upper lip mucosa between the bite block and the teeth led to mucosal ischemia, which could have worsened by Tc-electrical stimulation and led to this upper lip swelling. The use of conventional C3/C4 motor zone Tc-electrical stimulation ( $1 \mathrm{~cm}$ anterior to $\mathrm{C} 3 / \mathrm{C} 4$, over the motor cortex area, called M3/M4) for facial MEP monitoring causes stronger jaw clenching, because the electrodes are closer to the facial motor cortex, jaw muscle and trigeminal nerve. ${ }^{2}$ Both corticobulbar activations with pulse trains and direct trigeminal nerve stimulation can cause severe bite injury if the intraoral structures are caught between the teeth or between teeth and bite block. In this case, despite repeated stimulation (30 times), the patient did not get lip laceration, perhaps due to the less intense facial muscle contraction using M1/M2 Tc-electrical stimulation (1-cm anterior to C1/ C2, over the motor cortex area) as compared with M3/M4 zone $(1 \mathrm{~cm}$ anterior to $\mathrm{C} 3 / \mathrm{C} 4$, over the motor cortex area).
From our experience (unpublished data), medial stimulation (on the M1/M2) produces reliable responses with less movement and complications when compared with more laterally placed stimulating electrodes (M3, M4).

One should be careful while applying the bite block to ensure that the tongue, buccal mucosa, and lips are free from the bite block. Although frequent intraoperative checking of proper positioning is advocated, it is impractical during craniotomy and in patients undergoing surgery in a prone position. Appropriate size selection of the bite block is also important to prevent complications; an undersized bite block can cause tongue, oral mucosal and lip injury, while an oversized bite block can cause tongue folding as well as postoperative tongue swelling and airway compromise. Bilateral bite blocks using different materials (rolled gauze/gauze around the plastic syringe/using foley catheter and the glove/ silicone bite block) between the upper and lower molar teeth can also be used to prevent bite injuries. ${ }^{3}$ After placing the appropriate size bite block, checking whether the intraoral structures are free is mandatory. Proper placement of bite block should be added as a part of the surgical checklist in patients undergoing Tc-MEP monitoring to improve patient safety.

\section{Conflict of Interest}

None declared.

\section{References}

1 Tamkus A, Rice K. The incidence of bite injuries associated with transcranial motor-evoked potential monitoring. Anesth Analg 2012;115(3):663-667

2 Macdonald DB. Intraoperative motor evoked potential monitoring: overview and update. J Clin Monit Comput 2006;20(5):347-377

3 Sasidharan GM, Karre B. How to make a Do-it-yourself, Disposable bite guard using easily available materials to prevent tongue and lip injuries during motor evoked potential monitoring in Neurosurgery. Cureus 2019;11(8):e5536 